\title{
A Study of the Lubrication of EHL Point Contact in the Presence of Longitudinal Roughness
}

\author{
Johan Guegan $^{1} \cdot$ Amir Kadiric $^{1} \cdot$ Hugh Spikes $^{1}$
}

Received: 14 February 2015/Accepted: 13 May 2015/Published online: 4 June 2015

(c) The Author(s) 2015. This article is published with open access at Springerlink.com

\begin{abstract}
This work investigates the effect on elastohydrodynamic lubrication of roughness ridges oriented along the rolling-sliding direction, such as may be present on rolling bearing raceways. The roughness of the three specimens tested is characterised by the RMS of surface heights and a dominant wavelength. Optical interferometry and a ball-on-disc set-up were employed to map the oil film thickness. The paper first describes a novel procedure to carry out optical interferometry measurements on rough surfaces. Film thickness maps from the central part of the contact were obtained for a range of speeds in pure rolling and rolling-sliding conditions. The evolution of the film distribution with increasing speed along with the in-contact RMS and the real area of contact was calculated. The film maps show that the lift-off speed increases when roughness is introduced compared with smooth surfaces, while the average film thickness remains very close to the smooth case. The general horseshoe film shape that becomes visible at higher speeds is discussed. Using an inverse solution approach based on measured in-contact roughness, the pressure distribution is estimated in a rough, lubricated contact and its evolution with speed is explained. The findings provide important insights into the transition from boundary, through mixed, to full EHL lubrication for longitudinal roughness.
\end{abstract}

Johan Guegan

j.guegan11@imperial.ac.uk

1 Department of Mechanical Engineering, Tribology Group, SKF UTC for Tribology, Imperial College London, London SW7 2AZ, UK
Keywords Elastohydrodynamic lubrication - Mixed lubrication - Micro-EHL - Surface roughness - Inverse analysis · Pressure distribution

\section{Introduction}

Over the last few decades, machine elements such as bearings and gears have been subject to increasingly severe operating conditions. This has led to a progressive reduction in the lubricant film thickness down to values comparable to the roughnesses of the contacting surfaces. As a result, many elements are now operating for long periods of time in the mixed/boundary regimes in which the lubricant film is too thin to provide full separation of the surfaces so that a significant part of the load is born by the asperities. In some cases, it has been observed that a so-called microEHL film is produced at the top of the asperities [1-3].

Two types of approach have been used by previous authors to study the effect of roughness on elastohydrodynamic lubrication (EHL). The older is a stochastic approach where the roughness is described as a statistical distribution [4-6]. However, in the last 20 years most authors have adopted a deterministic approach in which idealised textures are considered. The experimental part of this work was made possible by the development of the optical interferometry technique introduced in the 1960s by Cameron and Gohar [7] for the measurement of lubricant film thickness in EHL contacts. Work on rough surfaces was then pioneered by Jackson and Cameron [8] with the study of scratches produced on a ball by a cutting tool. To date, a variety of surface features have been studied, both numerically and experimentally, including dimples or dents [9-12], bumps [13, 14] and ridges [15, 16]. These 
researches have enabled some fundamental mechanisms that govern the lubrication of rough surfaces to be determined.

An important aspect of this problem is how the amplitude of the roughness is reduced due to elastic deformation when it enters the loaded area. This has been extensively studied numerically for various cases [17-19] and observed experimentally [14]. Another important concept is the liftoff speed which is the speed at which the lubricant film becomes thicker than the highest asperity, providing full separation between the contacting surfaces. Hence, it is a good way to characterise the transition from the mixedlubrication regime to the full EHL regime. In particular, it was studied by Masen et al. [20] for a range of roughness produced with various manufacturing processes. It was found that the presence of roughness affected significantly the lift-off speed and also that it varied depending on the roughness considered. However, the experiments were performed using a capacitance/resistance technique which measures the probability of contact and does not allow a precise study of what happens at individual asperity, unlike the optical interferometry technique.

Providing that the undeformed topography and maps of film thickness are known, the displacement field and thus the pressure can be calculated by an inverse solution approach. Various authors $[21,22]$ have used this to find the pressure distribution in different contexts. The technique used in the current paper involves first calculating the influence coefficient matrix [23] which links the pressure and displacement fields. Then, as shown in Stanley and Kato [24] and Kadiric et al. [25], the problem can easily be inverted by converting it into the frequency domain.

The study of the effect of rough surfaces on lubrication is of particular relevance to rolling element bearings, where the balls are usually very smooth but the raceways are relatively rough. The machining processes used in the manufacture of rolling bearings can produce roughness structures that are dominated by ridges in the circumferential direction, i.e. parallel to the rolling/sliding direction. In this paper, as indicated in its title this form of roughness aligned along the rolling/sliding direction is termed "longitudinal".

In the current study, the specimens tested are AISI 52100 steel balls that have been artificially roughened with a cutting tool to obtain repeatable, circumferentially oriented roughness structures. A contact is produced by loading and rotating these specimens against a glass disc in a ball-on-disc set-up and measuring the lubricant film distribution within the contact using spacer layer-based optical interferometry [26]. Measurements were taken over a large range of speeds so that it was possible to observe the lubricant film build-up from boundary to full EHL. The objectives of this work were:
- to measure film thickness distributions up to high entrainment speeds, so as to span the full range of lubrication regimes for rough surface contact.

- to understand how different roughness parameters affect film distribution over the speed range.

- to estimate the pressure distribution and link it to the micro-EHL phenomenon that appears at the top of the roughness ridges.

\section{Experimental Techniques}

\subsection{Ball-on-Disc Rig}

A contact is produced in an EHL ball-on-disc rig in which a steel ball is loaded against a glass disc (see Fig. 1). The ball and the disc are driven by two independent motors so that it is possible to achieve mixed rolling-sliding as well as nominally pure rolling conditions. The lower surface of the disc is coated with a 20 -nm-thick semi-reflective chromium layer and a silica spacer layer of around $130 \mathrm{~nm}$ in thickness that enables very thin film measurements [26]. During tests, light is shone on the contact where approximately half of the incoming beam is reflected from the chromium layer, while the remainder travels through the spacer layer

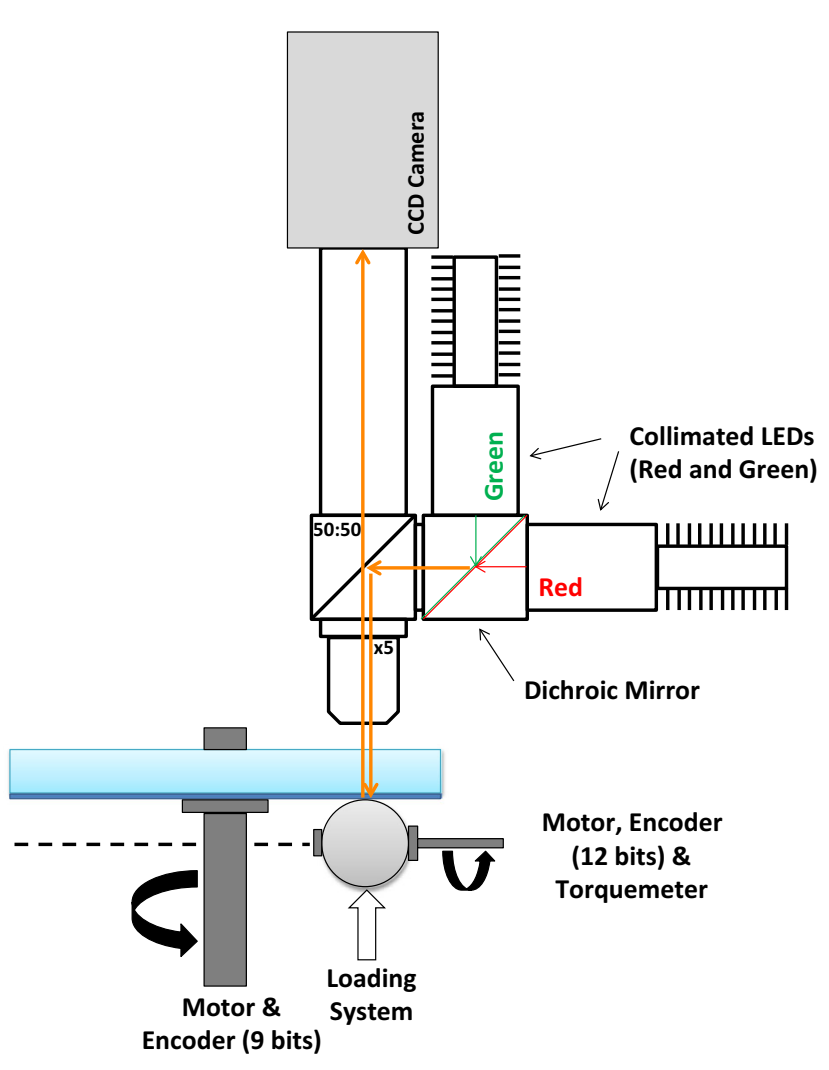

Fig. 1 Schematic of the set-up 
and the lubricant film before being reflected back by the surface of the ball. The difference in path length between the two parts of the incoming beam produces a difference in phase between them, which generates interference fringes when they recombine. The images obtained are interferograms of the contact which can be captured via a CCD camera. These interferograms can be subsequently analysed to generate maps of the lubricant film thickness.

\subsection{Technique for Measuring EHL Film Thickness in Rough Contacts}

The standard interferometry technique implemented on the ball-on-disc rig is designed to measure EHL film thickness in smooth contacts. To be able to study roughened ball surfaces, several modifications had to be made to the standard set-up. These are described below.

In order to avoid excessive blurring as roughness passes through the field of view, it is necessary to use a camera with fast shutter speed. This is not usually a problem with extremely smooth specimens. In this study, $800 \times 800$ pixel resolution interferograms were captured with a highspeed camera at a shutter speed of $10 \mu \mathrm{s}$. At $2.2 \mathrm{~m} / \mathrm{s}$, the surfaces travel 22 microns and in practice it has been found that above this speed the roughness became too blurred to be analysed in detail. The magnification of the lens was $5 \times$ so that each pixel covered an area of $1 \mu \mathrm{m} \times 1 \mu \mathrm{m}$ on the ball.

To monitor lubricant film build-up at precisely the same location on the ball as the speed was increased, and thus study the same roughness features, the camera was triggered off a fixed ball position for the duration of a test. To do this, a 12-bit encoder (4096 positions) was added to the ball shaft allowing a circumferential resolution around the ball of $15 \mu \mathrm{m}$ for a ball of diameter $19.05 \mathrm{~mm}$.

For accurate calculation of the lubricant film thickness, the spacer layer thickness must be known. This is not perfectly constant around the disc. During these tests, the camera is triggered from the ball position rather than from the disc position, as is normally the case, so the spacer layer thickness may vary from one image to another. To prevent this, the disc spacer layer thickness was mapped using a smooth ball by taking interference images all around the disc. A circumferential variation in the coating thickness of about $\pm 1 \mathrm{~nm}$ is usually observed. To eliminate the effect of this variation, the triggering system was set up so that the camera was triggered only when the exact selected roughness position on the ball was in contact with a portion of the disc lying in a tightly specified range of disc track within which the coating thickness variation was measured to be minimal. This "double triggering" also ensured that the image stayed focused since it minimised the effect of disc runout. The triggering set-up was validated using a smooth ball that had a $500-\mu \mathrm{m}$ lasertextured square at one position on the surface. Figure 2 shows images triggered on the ball position at different speeds. Over a large range of speed, it is clear that the square barely moves with respect to the triggering position and is not blurred, indicating that the triggering system is working as expected.

Some researchers have used white light $[2,27]$ to generate interference fringes for film thickness mapping. However, white light sources are usually broad and have a poor coherence length, typically around $500 \mathrm{~nm}$ for a halogen lamp, which means that the intensity of the interference fringes decreases rapidly with increasing film thickness and it becomes difficult to measure any film thicker than $500 \mathrm{~nm}$. To overcome this problem, more coherent light sources such as monochromatic LEDs or lasers can be used. In this study, a green LED $(530 \pm 15 \mathrm{~nm})$ and a red LED $(617 \pm 8 \mathrm{~nm})$ were used in combination. Such a duo-chromatic system has been employed in the past, for example by Wedeven [28] and Kaneta et al. [29], but has usually involved filtering a spectral lamp. Due to their high divergence, the LED light sources were collimated prior to being recombined with a dichroic mirror. The obtained light source has a coherence length of $2.8 \mu \mathrm{m}$ which enables measurement of lubricant films that are over a micron thick.

\subsection{Optical Analysis Technique}

When using optical interference techniques to map film thickness, two issues have to be addressed.

First, a correspondence between the lubricant film thickness and the colour intensity has to be determined. To do this, one of two different calibration methods is usually employed. One uses a static contact between a smooth ball and the coated disc. When the light is shone into the contact, Newton's rings are formed and the colour intensities are scanned radially and related to the film separation using either a contact model such as Hertz (see [27]) or the periodicity of the fringes (see [30]). The second calibration technique is explained in Choo et al. [31] and involves measuring central film thickness versus entrainment speed with a very smooth ball using ultrathin film interferometry [32] and then taking interference images over the same speed range to follow the evolution of the colour intensities in the central area. Ultrathin film interferometry is an absolute technique based on measurement of interference wavelength and does not require external calibration.

The second issue that has to be addressed is that, due to experimental error and the quasi-periodic nature of the calibration curve, it is possible for a given set of colour intensities to give several solutions for the film thickness. In practice, authors have either focused on film thickness 
Fig. 2 Images of a square textured surface passing through the field of view at different speeds

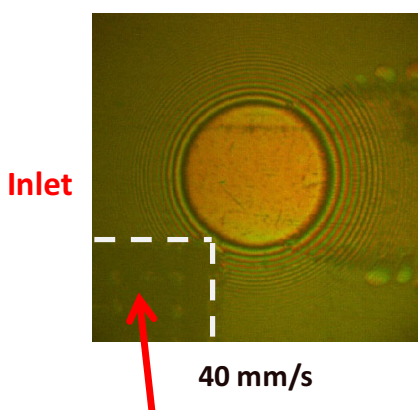

Laser Textured square

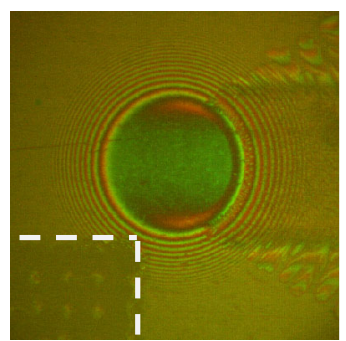

$200 \mathrm{~mm} / \mathrm{s}$

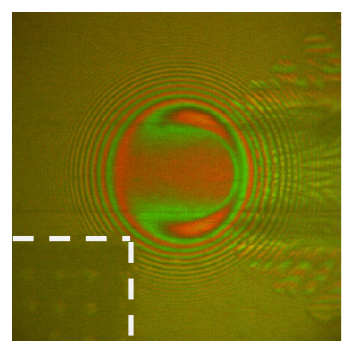

$1.1 \mathrm{~m} / \mathrm{s}$ values that fall in the first period of the calibration curve (i.e. below $250 \mathrm{~nm}$ ) or have defined a range of film thickness and chosen the best match within it.

All the above techniques give the correct intensity variation trend of the interference fringes against film thickness, but they also assume that the surfaces studied are as reflective as smooth surfaces. However, when rough surfaces are considered, it is observed that reflected light is diffused as opposed to the specular reflection of smooth surfaces. Hence, for a given film thickness the corresponding intensity of the interference fringes will be lower for rough than for smooth surfaces.

\subsection{Novel Procedure for Optical Measurements}

To enable the measurement both of very thick films and from rough surfaces, a novel procedure for film thickness measurement was developed. This involves two steps.

(a) Optical properties of the system: interference fringes period and initial phase shift

Similar to Molimard [27], a smooth ball is loaded against the disc in the presence of lubricant and the intensities of the circular red and green fringes are scanned radially as shown in Fig. 3.

Using Hertz theory, the gap outside of the contact is calculated and related to the intensity of the fringes. This is shown in Fig. 4.

As evident in Fig. 4, due to factors such as the loss of coherence of the light and the curvature of the ball, the amplitude of the fringes decreases with the increasing gap. To eliminate this effect, the relative optical interference intensity (ROII) can be used (see [33]). This is defined as:

$\bar{I}=\frac{2 I-I_{\max }-I_{\min }}{I_{\max }-I_{\min }}=\cos \left(\frac{4 \pi n h}{\lambda}+\Phi\right)$

where $I_{\max }$ and $I_{\min }$ represent the maximum and minimum intensities for each period of the curve. The effect of this transformation can be seen in Fig. 5 for the red fringes; the curve shown is a normalisation of the original curve by the adjacent minimum and maximum intensities and has a very similar form to a cosine function. The period of this curve is equal to the period of interference that would be produced by monochromatic light whose wavelength is equal to the average wavelength of the light source modulated by the camera's spectral response. The initial phase shift corresponds to the contribution of the spacer layer thickness and the phase shift due to the chromium layer.

From Fig. 5, it is apparent that the normalised intensity curve can be modelled by a cosine. Consequently, provided that the order of interference is known, the film thickness $h$ can be calculated using:

- If the intensity is decreasing on the period:

$$
h=h_{\text {peak }}+\frac{\lambda}{4 \pi n} \arccos (\bar{I})
$$

- If the intensity is increasing on the period:

$$
h=h_{\text {trough }}-\frac{\lambda}{4 \pi n} \arccos (\bar{I})
$$

The red and green channels can be used independently to calculate the film thickness and were found to give almost identical results. In this study, the film thickness is taken as the average of the film calculated using the two channels to reduce the experimental noise.

\section{(b) Image analysis}

For any particular point within the contact, the adjacent minimum and maximum intensities that need to be considered to calculate the ROII are not easily accessible. For example, when the surface is rough, the order of interference is not always known because of large variations in film thickness within the contact. Another parameter that may modify the amplitude of the fringes is the local orientation of the surface which is affected by the presence of asperities. Hence, a rough surface that is illuminated perpendicularly tends to diffuse the incident light, which reduces the amplitude of the interference.

To obtain the local values of the minimum and maximum intensities, a series of images is taken at different 
(a)

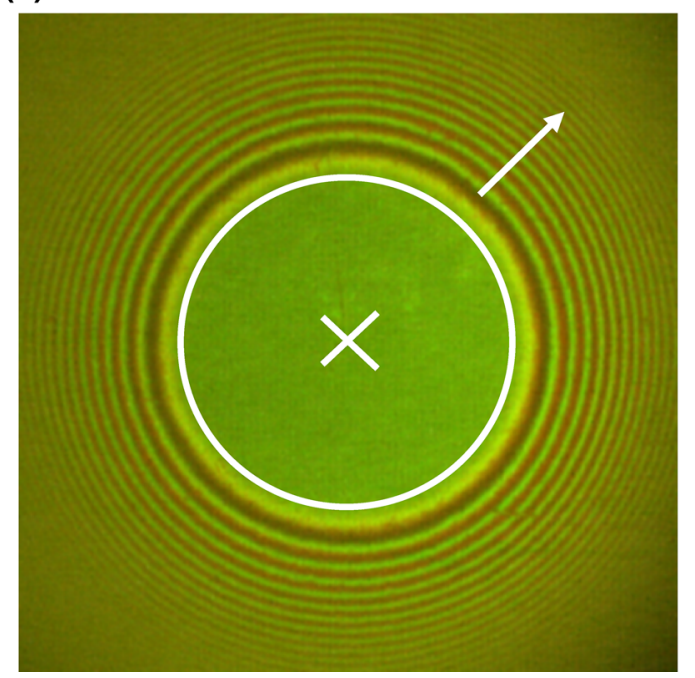

(b)

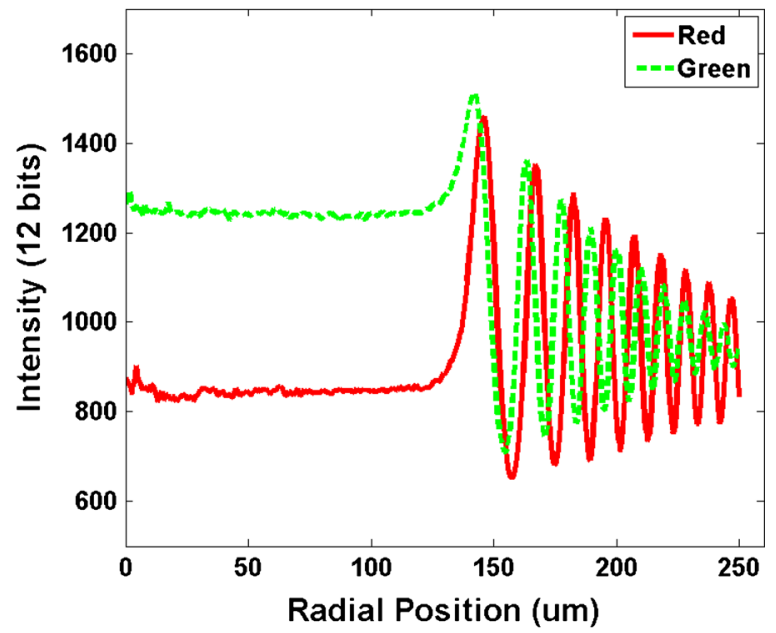

Fig. 3 a Interferogram showing a static ball-on-disc contact in the presence of lubricant, b intensity of the fringes

(a)

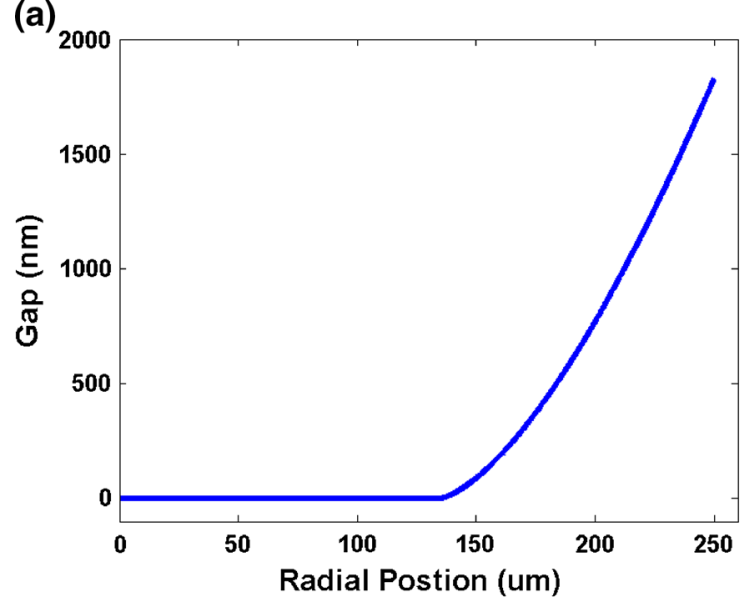

(b)

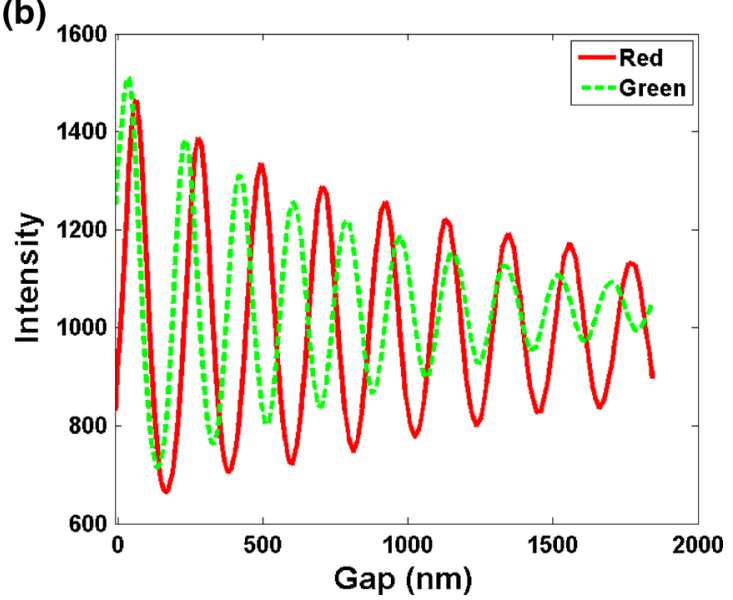

Fig. 4 a Gap calculated using Hertz theory, b intensity of the fringes versus gap, the amplitude of the waviness decreases with increasing gap

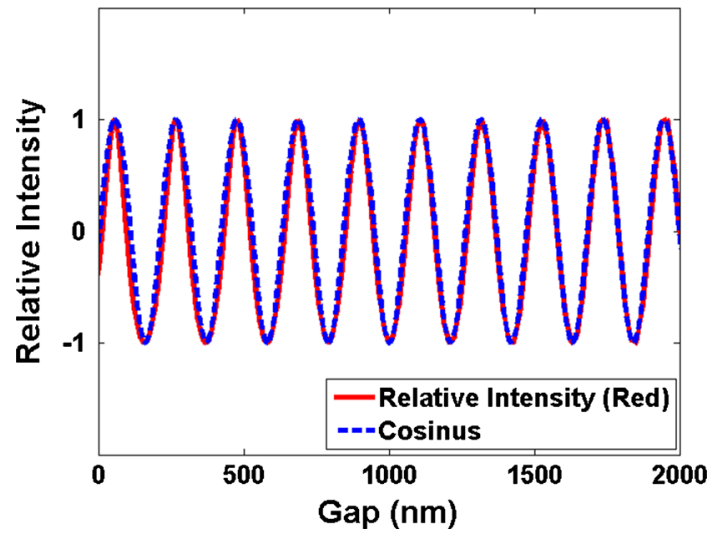

Fig. 5 Comparison between relative intensity of the fringes around a static contact and a pure cosine speeds, and the change in intensity of both colours is followed at each point. Assuming that the speed increase step is small enough that no order is missed, and that the film thickness monotonically increases with speed, it becomes possible to calculate the ROII everywhere. The high resolution of the ball shaft encoder and the fast shutter speed help to ensure that all images are taken near the same spot on the ball as illustrated in Fig. 2. However, despite these precautions, the contact tends to move by a few pixels from one image to another. To correct for this, a correlation algorithm is used to realign the images before analysis.

All the images are then stacked and the evolution of the red and green intensities against speed is observed at every 
point independently. The ROII is then calculated for each point of the contact at every speed following the method described previously. Figure $6 a, b$, respectively, show an example of recorded red and green intensities and the calculated ROII.

Using these data and Eqs. 2 and 3, it becomes possible to calculate quite reliably the film thickness for each speed everywhere in the contact (Fig. 5a). Equally, at each speed considered, a map of film thickness can be obtained. An example of calculated film thickness at a selected point and a complete film thickness map are shown in Fig. 7a, b, respectively.

Unlike in smooth contacts, in rough contacts considered here there is no one single value of minimum or central film thickness. In order to be able to extract representative values of film thickness from the obtained maps, a rectangular area equivalent to the flat central area of the smooth contact is selected inside the contact, avoiding the constriction. Maps are generated for a range of entrainment speeds, and then the following values are calculated:

- Minimum film thickness: average of the lowest $1 \%$ of points. This value is taken to represent the film thickness at the tops of the asperities.

- Maximum film thickness: average of the highest $1 \%$ of points. This value is taken to represent the film thickness in the valleys between the asperities.

- Average film thickness.

- RMS roughness of the separation: this is calculated as the standard deviation of the film distribution.

- Solid-to-solid contact: percentage of the contact area that is "in contact", determined as the points where the film thickness is measured to be $0 \mathrm{~nm}$.

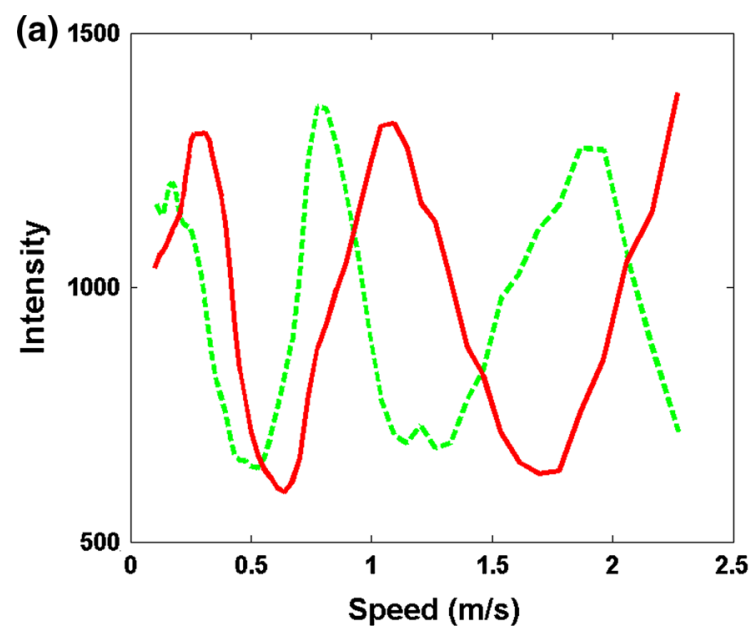

\subsection{Experimental Protocol}

The disc, ball specimens, ball shaft, ball carriage and lubricant pot are consecutively soaked in toluene and isopropanol. They are then dried with a heat gun in order to eliminate any trace of solvent.

The pot is filled with the test lubricant and left for 30 min in order to allow the set temperature (disc + ball specimen + oil) to stabilise. A particular ball position is chosen from which all the measurements of film thickness are subsequently taken. The position of the disc is also logged to enable the partial triggering.

\subsection{Test Parameters and Specimens}

The specimens are drilled AISI 52100 steel balls with a diameter of $19.05 \mathrm{~mm}\left(3 / 4^{\prime \prime}\right)$. The geometry and the material properties are summarised in Table 1.

Smooth balls and discs had RMS roughnesses of 20 and $5 \mathrm{~nm}$, respectively. Roughened balls with three different roughness structures were tested in this study. The textures were produced by careful roughening of the ball on a lathe to achieve periodic circumferential ridge-valley texture (parallel to the rolling/sliding direction). By varying parameters such as the feed rate and the radius of the cutting tool, it was possible to obtain surfaces with different dominant wavelength and peak-to-valley heights. The topographies of the three selected roughnesses are shown in Fig. 8.

Using a dedicated jig with angular positioning, it was possible to precisely locate the roughness and then capture the film thickness in this position. The measured roughness properties of the three selected rough specimens are listed in Table 2.

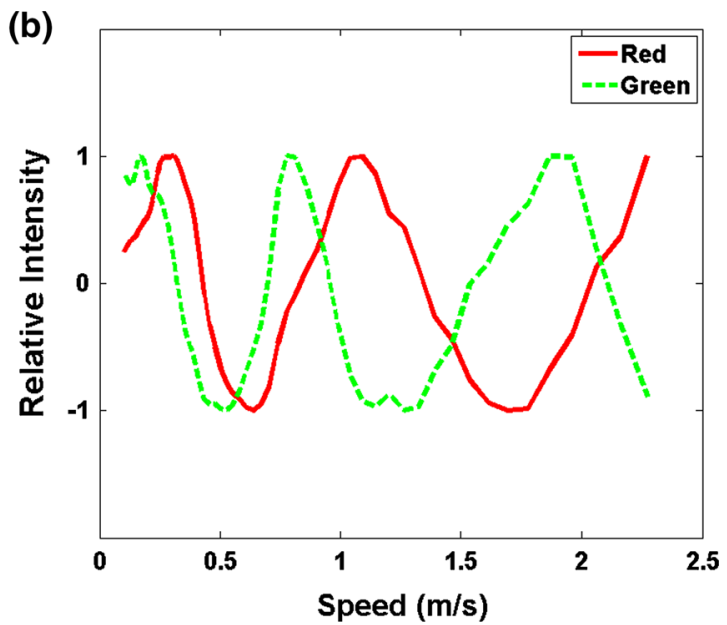

Fig. 6 a Variation in the intensity of the red and the green with speed for a particular point in a rough contact, b calculated ROII (Color figure online) 

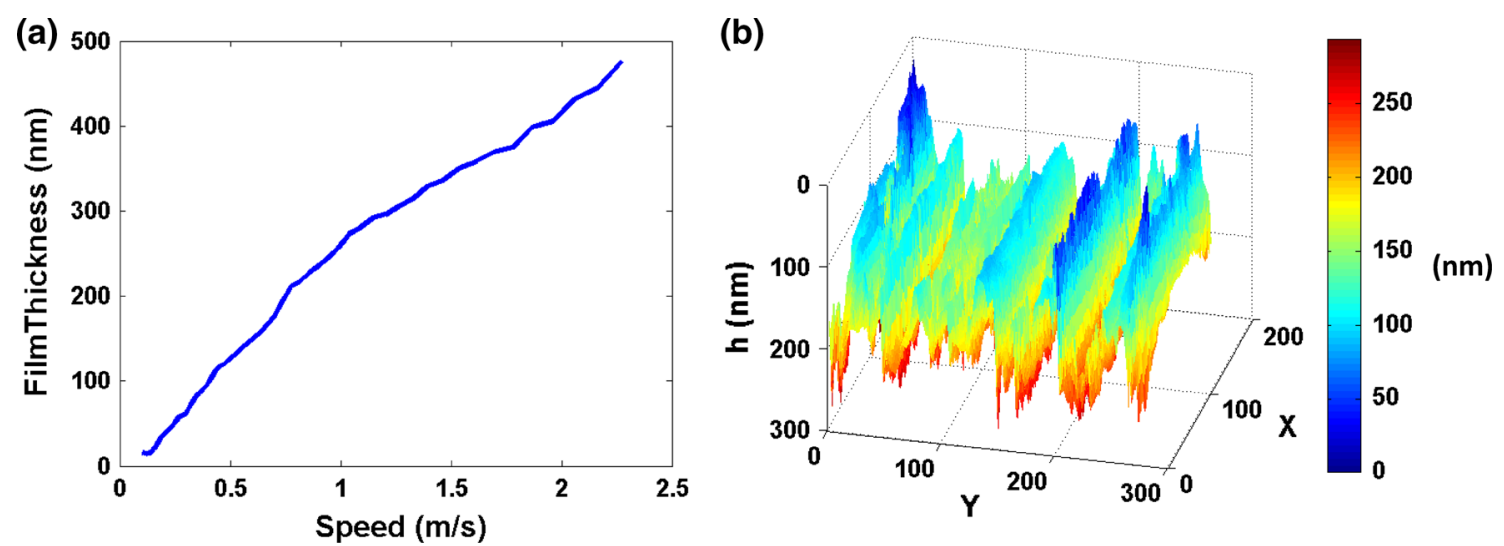

Fig. 7 a Film thickness against entrainment speed at one point in the contact, b example map of film thickness of a lubricated rough contact at a particular speed

Table 1 Geometry and material properties

\begin{tabular}{lll}
\hline Ball diameter & Young's modulus & Poisson's ratio \\
\hline $19.05 \mathrm{~mm}\left(3 / 4^{\prime \prime}\right)$ & Steel ball: $207 \mathrm{GPa}$ & Steel ball: 0.3 \\
& Glass disc: $75 \mathrm{GPa}$ & Glass disc: 0.22 \\
\hline
\end{tabular}

The specimens were tested in the ball-on-disc rig at the conditions listed in Table 3 .

In all the tests, a load of $20 \mathrm{~N}$ was applied, giving a nominal Hertz pressure of $0.527 \mathrm{GPa}$ and a contact diameter of $269 \mu \mathrm{m}$ based on smooth surface calculation. In the case of rough surfaces and this film conditions, the load is mostly born by the asperities so the local pressure is expected to be higher. The overall size of the contact zone did not seem to be significantly affected by roughness, suggesting that the important parameter controlling this was the radius of curvature of the ball and not of the asperities. In each test, the entrainment speed was increased in steps while maintaining a fixed slide-to-roll ratio (SRR), where the entrainment speed is the mean speed of the surfaces while the SRR is defined as:

$\mathrm{SRR}=\frac{U_{\text {Disk }}-U_{\text {Ball }}}{U_{\mathrm{e}}}$
Specimen 1
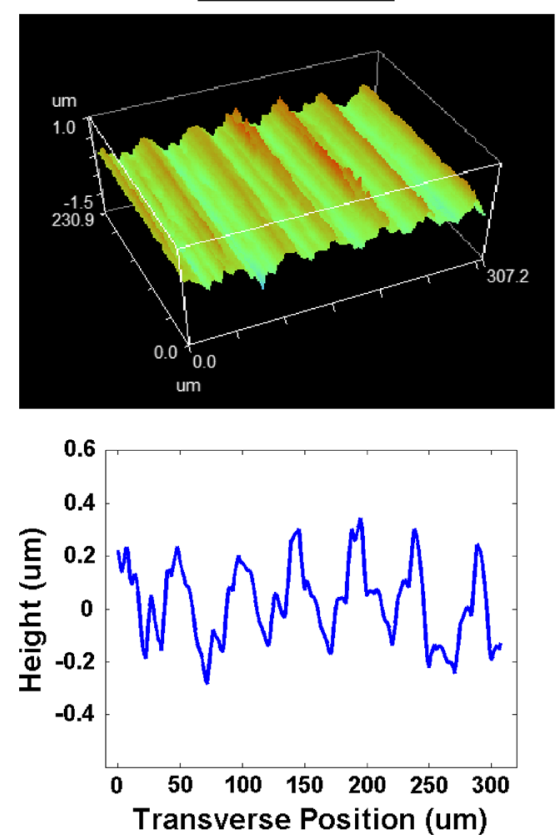

Specimen 2
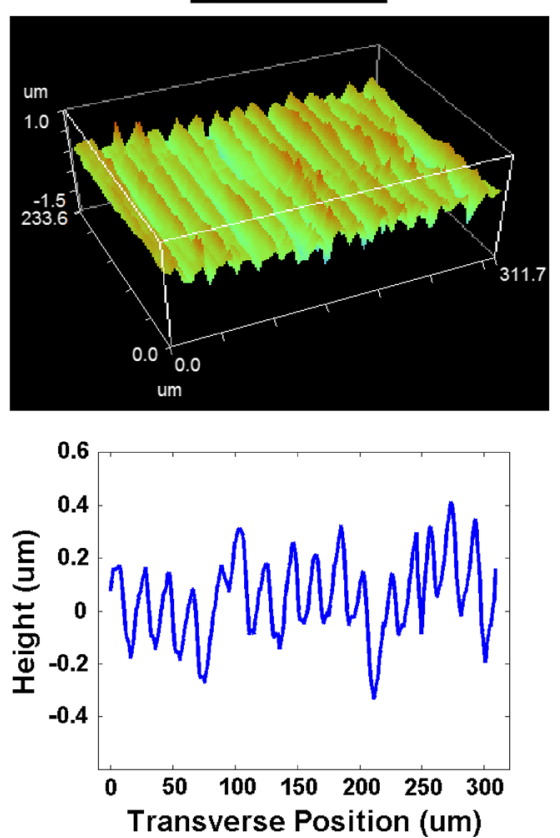

Specimen 3
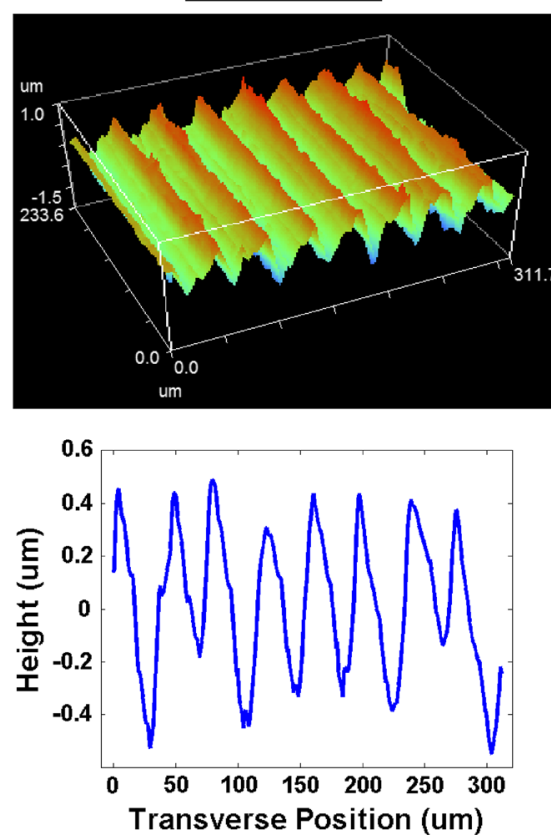

Fig. 8 Surface profiles of the three selected specimens as measured with a white light interferometer 
Table 2 Roughness parameters of the different specimens extracted via FFT

\begin{tabular}{llll}
\hline & Average peak-to-valley height $(\mu \mathrm{m}): \mathrm{H}$ & Dominant wavelength $(\mu \mathrm{m})(\mathrm{FFT}$ analysis $)$ & RMS $(\mu \mathrm{m})$ \\
\hline Specimen 1 & 0.52 & 45 & 0.15 \\
Specimen 2 & 0.49 & 19 & 0.15 \\
Specimen 3 & 0.97 & 39 & 0.27 \\
\hline
\end{tabular}

Table 3 Experimental conditions

\begin{tabular}{llll}
\hline Temperature & Load & Speed range & SRR \\
\hline $40.0 \pm 0.5{ }^{\circ} \mathrm{C}$ & $20 \mathrm{~N}$ & $0.02-2 \mathrm{~m} / \mathrm{s}$ & 0 and $50 \%$ \\
\hline
\end{tabular}

In all tests, the SRR was positive, i.e. the disc surface moved faster than the ball surface.

The oil tested was Shell Turbo 68, a Group II base oil with antioxidant additives, whose properties were measured and are listed in Table 4 . The test temperature was $40{ }^{\circ} \mathrm{C}$.

This lubricant and temperature were chosen to enable a full span of the lubrication regimes over the chosen entrainment speed range using just one oil. As seen in Fig. 9, for a smooth ball, film thickness varied from $10 \mathrm{~nm}$ (boundary conditions) to $1000 \mathrm{~nm}$ (fully flooded conditions) over the speed range available.

\subsection{Inverse Solution Model}

As shown in Molimard et al. [22], when accurate film thickness maps are available it is possible to use them as inputs to estimate the pressure distribution, assuming the undeformed surface is known. In our case, this requires the out-of-contact topography to be measured using white light interferometer (WLI) and matched to precisely the same location from which the film thickness maps are obtained in the ball-on-disc rig. To achieve this, an indent was made on the ball using a diamond cone (Rockwell $\mathrm{C}$ indenter). Figure 10 shows an interference image of a static rough contact and a corresponding surface topography map observed under the WLI. It can be seen that the two surfaces are closely aligned.

To estimate the pressure distribution, the elastic displacement map of the surfaces is needed. This is the difference between the loaded and the unloaded surface topographies plus a penetration value, the approach of

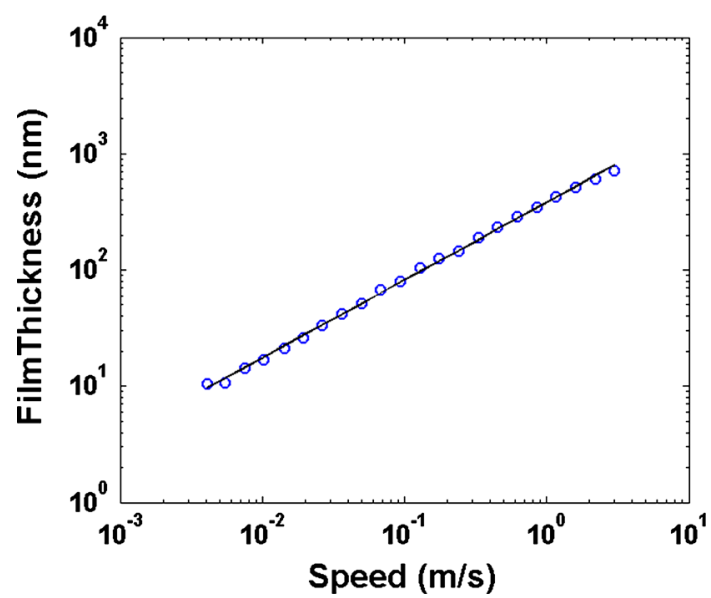

Fig. 9 Film thickness versus entrainment speed for the test oil and smooth specimen at $40 \mathrm{C}$

centres of the two bodies. The latter is an unknown constant, and to obtain it, the displacement was approximated as Hertzian outside of the contact area and the constant was found by determining at the difference between the two solutions at the edge of the contact. Figure 11 shows the transverse profile of the displacement field before and after this operation. One disadvantage of this method is that it assumes that the pressure outside of the contact is zero. This is valid for static and low speed contacts, but is not the case in the inlet of lubricated contacts at high speeds. To evaluate the inlet pressure, it is possible to extrapolate the film profile around the contact and this was explored, but was found not to significantly affect the calculated pressure distribution within the contact area. Thus, it was not implemented in the results shown in this paper.

The pressure field $\boldsymbol{P}$ and the displacement field $\boldsymbol{W}$ are linked by the influence coefficient matrix $\boldsymbol{K}$ which is a function of the material properties and the dimensions of the mesh [23].

Table 4 Oil properties

\begin{tabular}{llll}
\hline & Viscosity at $40{ }^{\circ} \mathrm{C}(\mathrm{cP})$ & Viscosity at $100{ }^{\circ} \mathrm{C}(\mathrm{cP})$ & Refractive index at $40{ }^{\circ} \mathrm{C}$ \\
\hline Shell Turbo 68 & 57.7 & 7.1 & 1.469 \\
\hline
\end{tabular}


Fig. 10 a Static contact as seen in the ball-on-disc set-up showing an indent, b same location observed under WLI
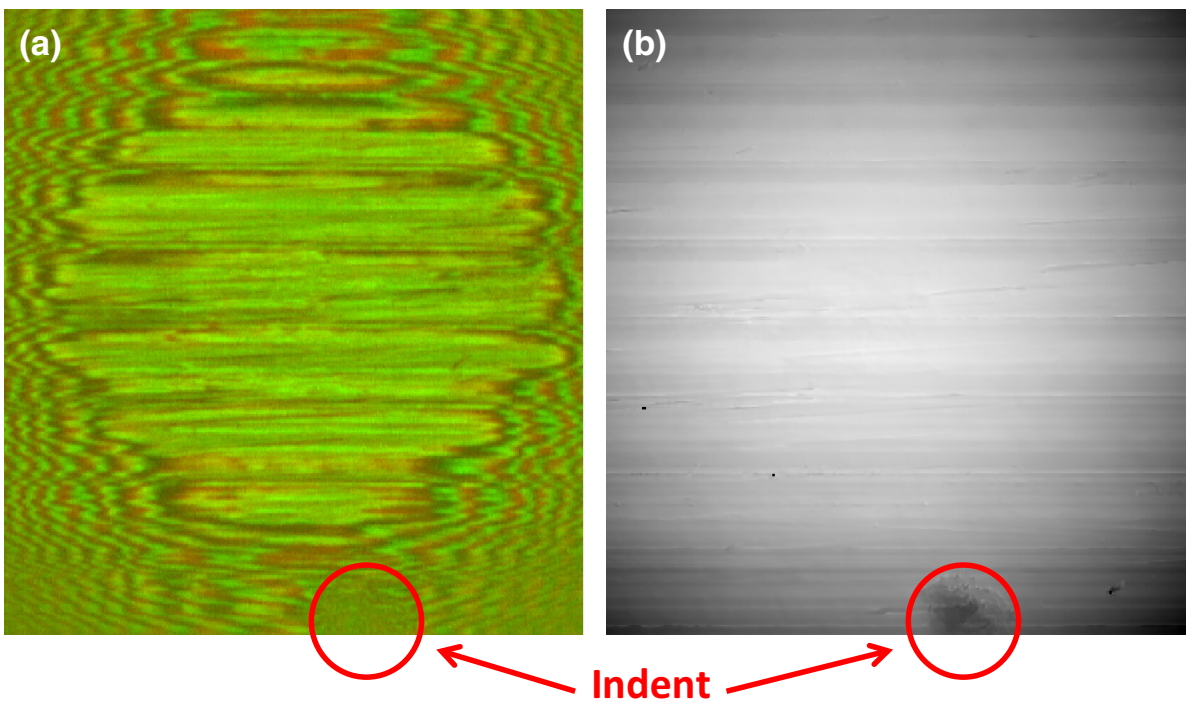

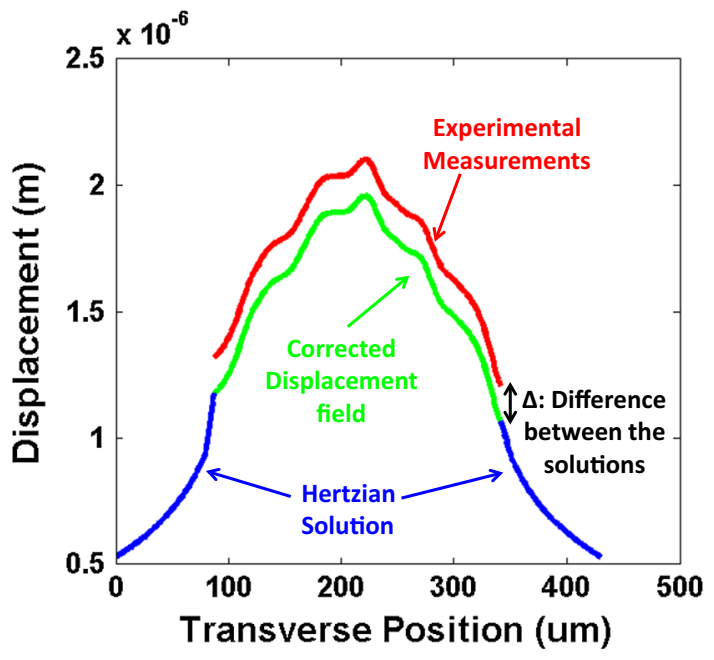

Fig. 11 Illustration of the procedure to calculate the displacement field

$W=\operatorname{conv}(K, P)$

where conv is the convolution operation. The problem can easily be inverted by considering the fast Fourier transform (fft) of the matrices:

$P=\operatorname{ifft}\left(\frac{\mathrm{fft}(W)}{\mathrm{fft}(K)}\right)$

where ifft is the inverse fast Fourier transform that enables conversion into the spatial domain.

As well as the predominant longitudinal ridge texture, the ball surfaces also had much finer roughness features, predominantly along the rolling direction, with wavelength ca 1-5 $\mathrm{mm}$ and amplitude $10-20 \mathrm{~nm}$. Since the focus of this study was the impact of the ridges on pressure, the displacement was smoothed prior to being used as input to calculate the pressure distribution. To do so, the macrocurvature was first extracted using a discrete cosine transform, and then the result was smoothed with a simple averaging. The kernel used was a 9 by 9 points square window, which represented a $9 \mu \mathrm{m}$ by $9 \mu \mathrm{m}$ area. This was found to be adequate to smoothen the lower frequencies without excessively attenuating the higher frequencies. Yet it was observed that the smoothing led to a slight reduction in the ridge amplitude; to compensate for this, the profile was multiplied by a constant. Finally, the macro-curvature was re-added to the profile.

\section{Experimental Results}

\subsection{Film Distribution}

During a test, the speed was slowly increased and images were taken in the same position on the rough ball. Figures 12 and 13 compare interferograms from a lubricated contact of a smooth specimen and rough specimen 1 in pure rolling at six entrainment speeds. It is evident that as the entrainment speed increases the colour changes, indicating lubricant film build-up. In the rough specimen case, the changes in colour occur faster in the valleys between the asperities, showing that the film build-up primarily occurs in these regions while the tops of the asperities remain in contact.

As the speed increases, the horseshoe shape becomes more and more visible in Fig. 13. Figure 14a shows the transverse profile of film thickness for a selected set of speeds for specimen 1. At low speeds, the surface features are very compressed, leading to an almost conformal contact. As the speed increases, the lubricant film thickness 
Fig. 12 Interferograms of a smooth specimen at a range of entrainment speeds in pure rolling conditions
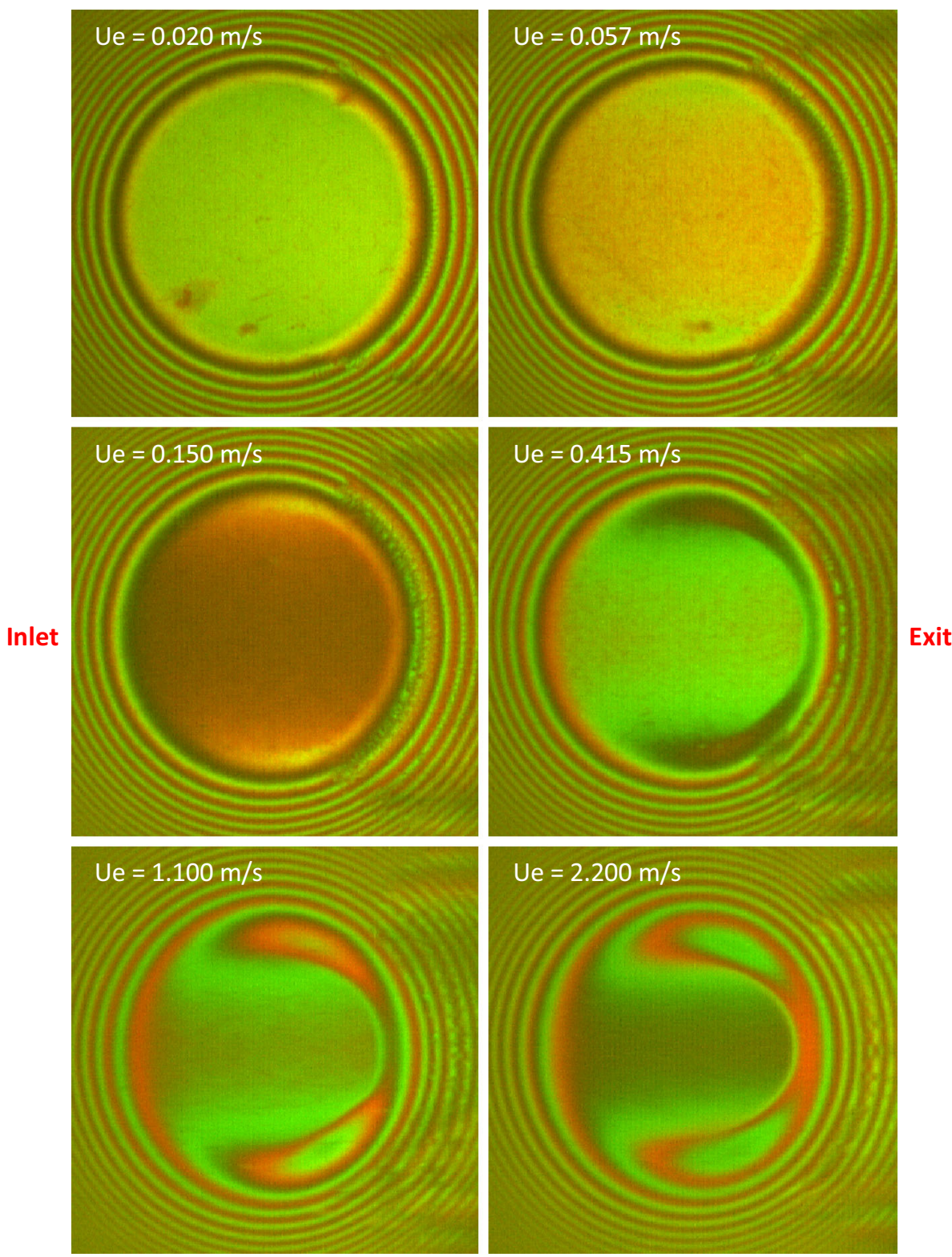

increases and the asperities start to recover. For comparison, the film profile for a smooth case at $1.1 \mathrm{~m} / \mathrm{s}$ was added to the chart. This shows that the film shape in smooth and rough cases follows the same general trend. The profile in the rough case varies around a centreline that is well represented by the smooth profile. In the centre of the contact area, the asperities are more squashed than at the edge. This is similar to what happens in the smooth case, where the deformation is larger in the centre.

At the top of the ridges, a micro-EHL phenomenon becomes visible. A ridge was selected from the interferograms as shown in Fig. 13, and the evolution of the average film thickness profile along the top of this ridge is shown in Fig. 14b. In this figure, the contact inlet is to the left and the exit to the right.

\subsection{Average Film Thickness Measurements}

As shown in Fig. 13, a square area was selected on each interferogram and the mean values described in Sect. 2.4b were calculated. The area of analysis was chosen to avoid the constriction region so that the measurements could be compared to the central film thickness region measured on a smooth specimen. The specimens were tested under both pure rolling and rolling-sliding conditions at $\mathrm{SRR}=50 \%$ over a range of entrainment speeds varying from 0.02 to $2.2 \mathrm{~m} / \mathrm{s}$. Figure 15 shows the minimum, the maximum and the average film thicknesses (as defined in Sect. 2.4b) over the selected area in the case of rough specimen 1 . The central film thickness measured with a smooth specimen 
Fig. 13 Interferograms of rough specimen 1 at a range of entrainment speeds in pure rolling conditions
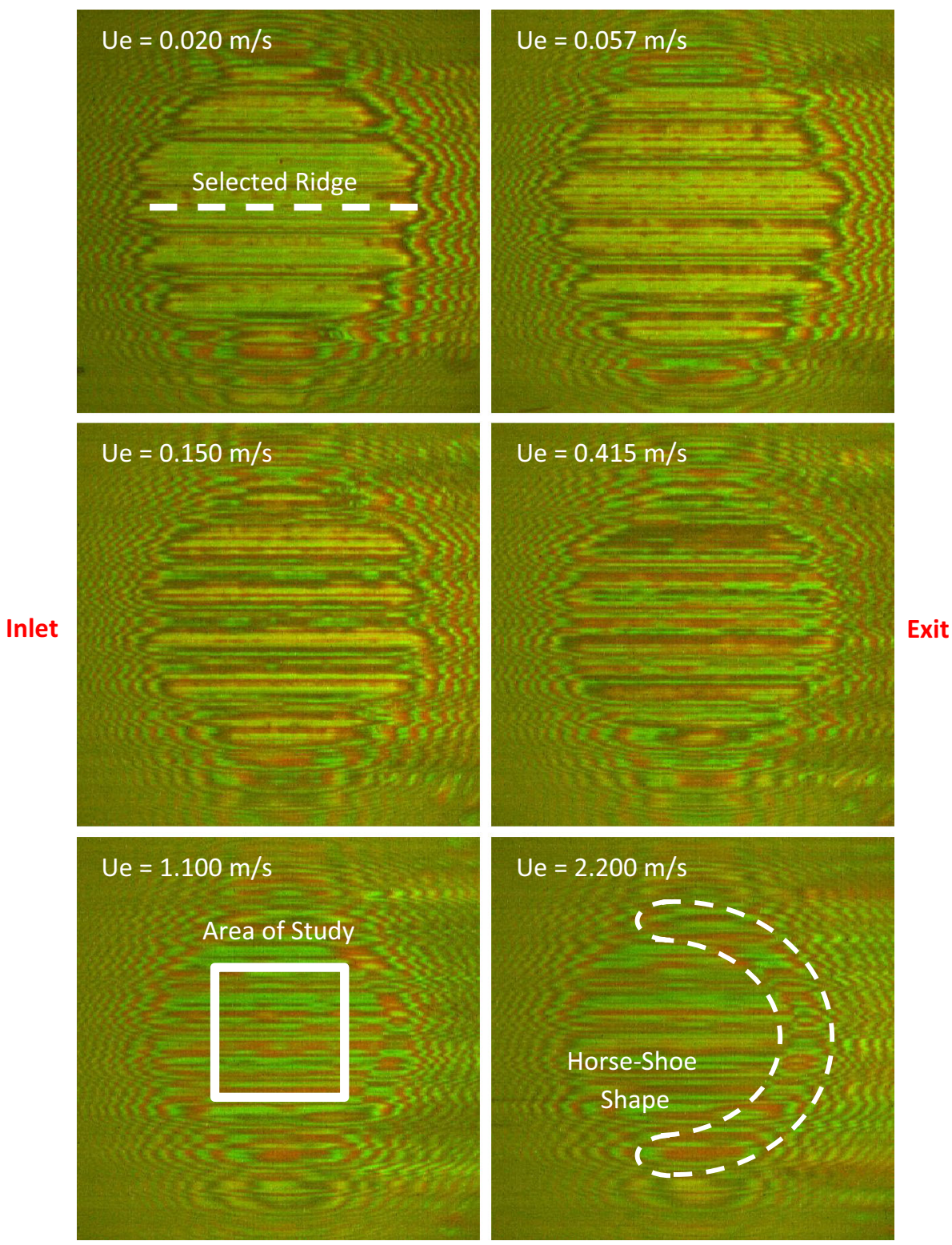

under the same conditions is also plotted for comparison. It is evident that there is very little difference between the nominally pure rolling and $50 \%$ sliding cases.

The minimum and maximum film thicknesses in pure rolling for the three different rough specimens are shown in Fig. 16; the average film thickness is not shown because it was very similar for all the roughnesses and is well represented by the smooth surface curve. The three rough specimens all show similar trends:

- The minimum film thickness remains zero up to a critical speed, the "lift-off" speed.

- The average film thickness is very similar to the central film thickness measured with a smooth specimen in the same conditions.
However, it can be seen in Fig. 16 that, over the range of conditions tested, the surface separation (minimum film thickness) increases at a lower rate for the roughest specimen (specimen 3, RMS $=0.27 \mu \mathrm{m}$ ) compared with the smoother specimens ( 1 and $2, \mathrm{RMS}=0.15 \mu \mathrm{m}$ ). The wavelength of the roughness seems to have a marginal impact as the minimum and maximum film thicknesses measured with rough specimens 1 and 2, which have a 40 and $20 \mu \mathrm{m}$ wavelength, respectively, but almost the same RMS.

Using the film thickness maps in the selected area the RMS of the separation was calculated. The practical significance of this RMS value is discussed later in this paper. Figure 17 shows how the ratio between this in-contact RMS and the undeformed RMS measured outside of the 

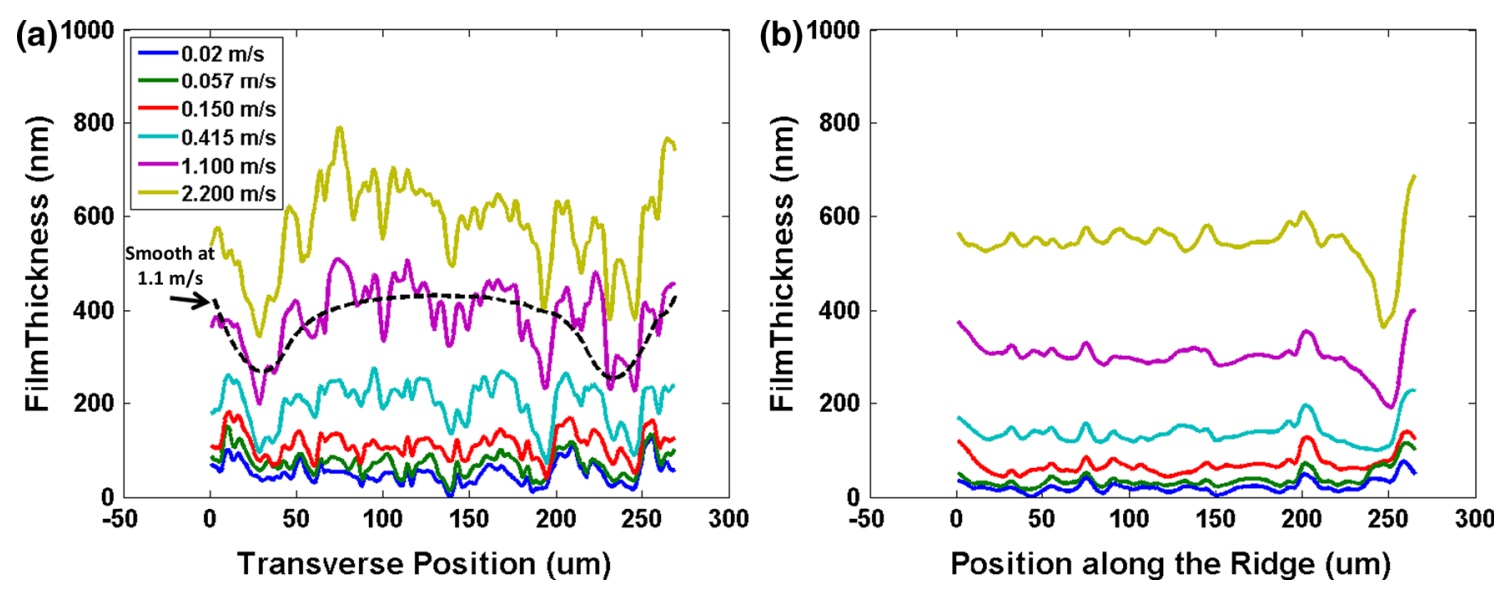

Fig. 14 a Film thickness profile in the transverse direction for rough specimen 1, b film thickness profile along the selected ridge at different speeds for specimen 1. Pure rolling conditions

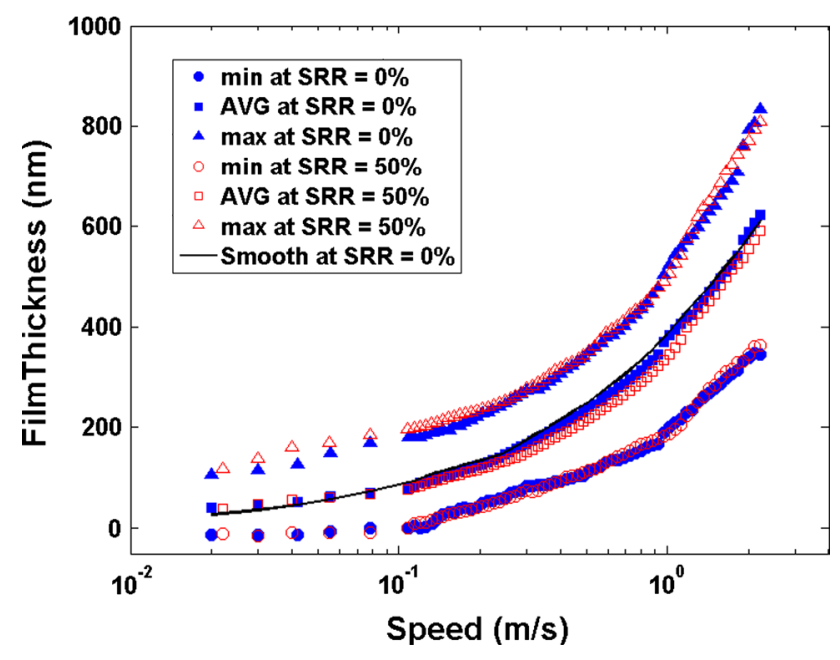

Fig. 15 Film thickness versus entrainment speed for rough specimen 1 in pure rolling and rolling-sliding conditions

contact is affected by an increase in the entrainment speed. While it is greatly reduced at low speed compared with the unloaded out of the contact RMS, lubricant film build-up enables the roughness to partially recover at higher speeds. The RMS of the two specimens with the same undeformed RMS recovers at approximately the same rate, while the roughest specimen recovers more slowly.

Lift-off occurs when there is no more solid-to-solid contact. To characterise the speed at which this occurs, it is appropriate to look at the real contact area (solid to solid) rather than the minimum film thickness. This is defined as the number of points where the film is zero (or appears negative due to local spacer layer compression). Figure 18 shows the evolution with speed of the fractional real area of contact over the area analysed. For each rough surface, the real area of contact falls to zero at a quite well-defined entrainment speed.

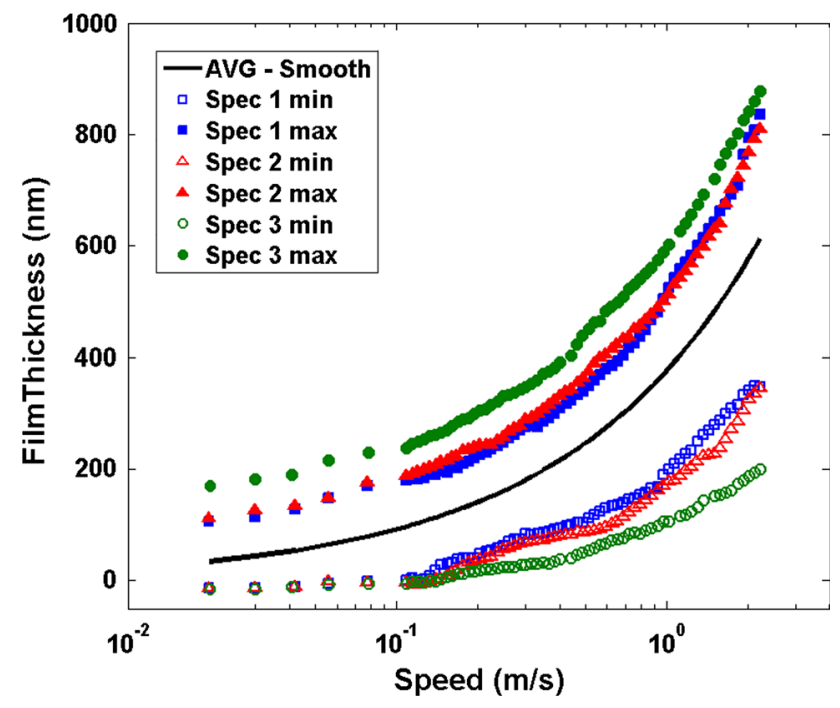

Fig. 16 Comparison of the minimum and maximum film thicknesses for the different specimens in pure rolling conditions

At low speeds, the rough specimen with the smallest wavelength (specimen 2) has the largest solid-to-solid area of contact. Specimen 1, with the same roughness but a higher wavelength, has a slightly smaller area of contact, while the roughest specimen has an even smaller area of contact. As the entrainment speed increases, the surfaces start to separate and the solid-to-solid contact diminishes.

However, this diminution evolves at a different rate depending on the specimen considered. As with the film thickness measurements, the two rough specimens with the same RMS show lift-off occurring at about the same speed, between 0.150 and $0.20 \mathrm{~m} / \mathrm{s}$, while the roughest specimen lifts off more slowly and there is still solid to solid up to $0.50 \mathrm{~m} / \mathrm{s}$. 


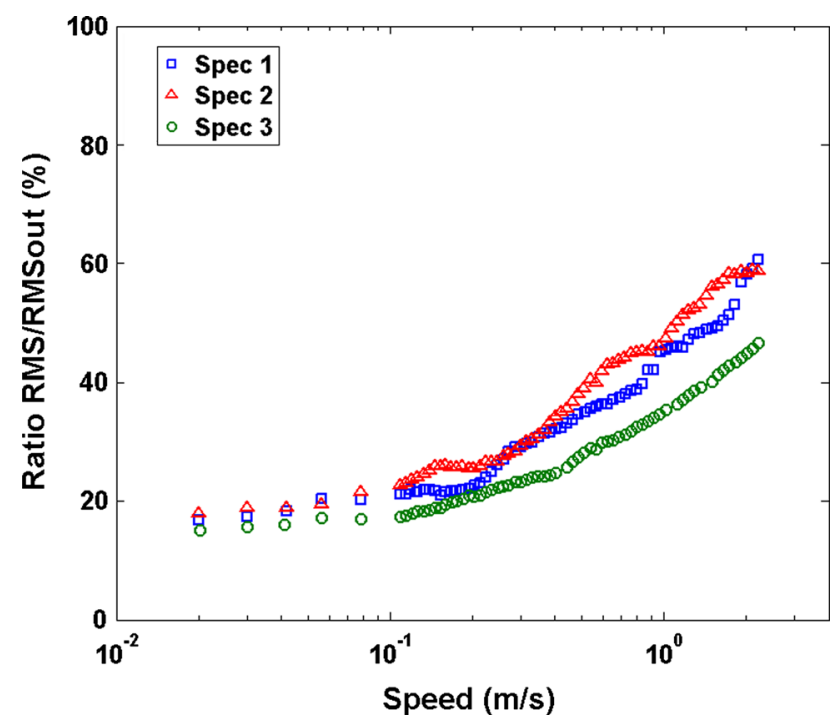

Fig. 17 Variation in entrainment speed of the ratio between the RMS inside the contact and the unloaded RMS in pure rolling conditions

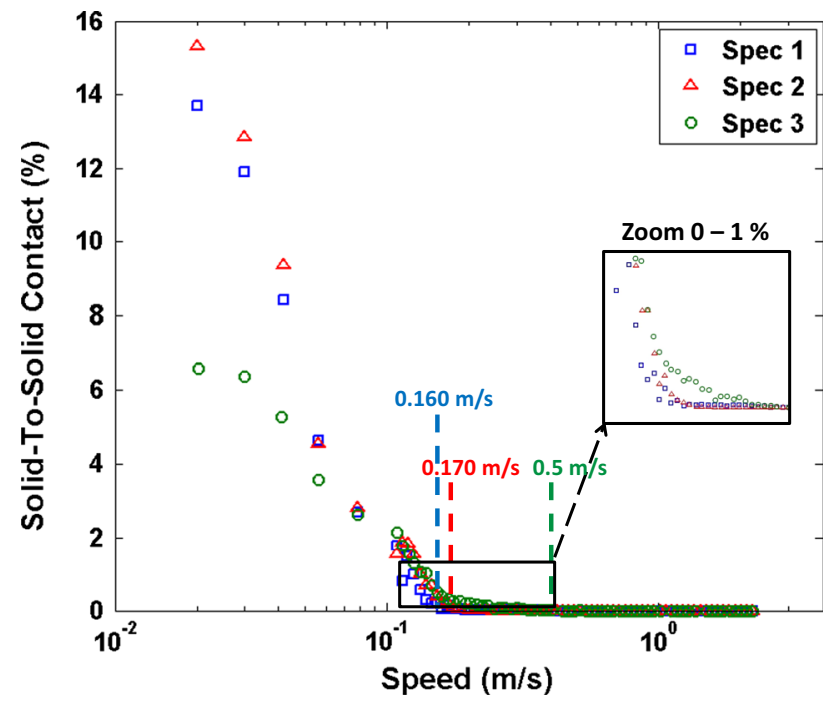

Fig. 18 Evolution of the solid-solid contact with entrainment speed in pure rolling conditions. The respective lift-off speeds are indicated for each specimen

\section{Discussion}

\subsection{Film Shape}

As was observed in previous work [8, 16], at low lambda ratio the roughness dramatically affects the film distribution. For example, at $0.150 \mathrm{~m} / \mathrm{s}$, the constriction is well defined in the smooth case but indistinguishable for rough specimen 1 . At this low speed, the asperities are apparently much squashed and the conformity of the surfaces (seen in Fig. 14a) implies that they bear a large part of the load. As the speed increases, the lubricant film increases in the valleys, the roughness starts to recover and the real area of contact is reduced.

At higher speeds (over $0.5 \mathrm{~m} / \mathrm{s}$ ), contrary to what has been observed in other studies [16], a horseshoe shape becomes visible. This difference from previous work can be explained by the higher speeds reached and the higher viscosity of the lubricant employed in the current study, which leads to much thicker nominal films. It is clear from the interferograms of Figs. 13 and $14 \mathrm{~b}$ that as the film becomes thicker a constriction appears at the exit of the contact. While micro-EHL features are particularly visible at the top of the ridges, a macro-horseshoe shape can also be seen over the contact as a whole. In Fig. 14a, the transverse film profiles are shown for rough specimen 1 along with the profile of a smooth ball at $1.1 \mathrm{~m} / \mathrm{s}$. As the speed increases, the asperities partially recover; however, the macro-curvature of the surface (i.e. when the roughness is ignored) follows closely that of the equivalent smooth case: the ridges in the central area are more compressed (more flattened), and a film reduction occurs at both edges of the contact (horseshoe). The rough contact behaves as if the roughness has been added to the smooth case deformation. Thus, the deformation seems to be far more influenced by the macro-geometry (i.e. the curvature of the ball) than the roughness.

The rough specimens were also tested in rolling-sliding conditions with a positive SRR equal to $50 \%$. Over the range of conditions tested, the measured film thicknesses were the same, within experimental error, as the ones measured in pure rolling conditions, suggesting that this level of sliding does not have any impact on the film distribution. This is important since it suggests that pure rolling behaviour is representative of that likely to be present in most rolling bearing contacts where SRRs are generally low. It also has implications on damage accumulation mechanisms in rough rolling-sliding contacts, such as micro-pitting, as it suggests that any increased asperity interaction at higher sliding speeds is not due to film thickness reduction.

\subsection{Influence of the Roughness Parameters on Film Build-up}

For all of the rough specimens, the minimum film thickness in the central region was significantly thinner than the central film thickness measured with a smooth specimen, as can be seen in Fig. 16. This can be explained by the gradient of pressure between the ridges and the valleys: at low speed, the higher pressure at the ridges forces the lubricant laterally into the valleys, where the pressure is lower. For rough specimens, the lift-off (i.e. when the surfaces fully separate) occurred at much higher speed than for smooth surfaces. It can be seen in Fig. 18 that for specimens 1 and 
2 , it starts at around $0.170 \mathrm{~m} / \mathrm{s}$, at which speed the film thickness in the central region is already above $100 \mathrm{~nm}$ for the smooth ball. Rough specimens 1 and 2 differ only in the wavelength of their roughness; they have the same RMS roughness and peak-to-valley height. Figure 16 shows that the maximum and minimum film thicknesses for the two specimens are very similar at all speeds. Specimen 3, which has greater RMS and peak-to-valley height, behaves differently in that the maximum film thickness is higher since valleys are deeper, and the surface separation increases more slowly with increasing entrainment speed. As the real contact area shows in Fig. 18, some residual contact remains up to a speed of $0.5 \mathrm{~m} / \mathrm{s}$. A very interesting result was that average film thickness in the central area was found to be reasonably close to the average film thickness, independent of the roughness considered.

All of the above suggests that the film build-up is only affected by the RMS of the surface and not significantly by the wavelength of the roughness for the range of roughness structures studied here.

\subsection{Roughness Recovery}

Recovery of the roughness can be estimated by the RMS of the separation. It should be noted that the observed RMS is the composite roughness of the contacting surfaces. As the glass disc has much lower elastic modulus than the steel, it can be assumed that the observed reduction in roughness is almost entirely due to smooth glass surface being deformed as the ridges elastically indent it, rather than the steel asperities flattened. Roughness recovery is then due to the indentations in the glass being reduced as the rough steel surface lifts away from it due to the build-up of film pressure.

At low speeds, the effective roughness is reduced to below $20 \%$ of the undeformed roughness. As the speed increases, the roughness partially recovers which shows that a growing part of the load is borne by the lubricant film.

The lift-off speed is the speed at which the load stops being supported in part by solid-to-solid contact and becomes wholly supported by hydrodynamic lift from the lubricant film. Since the contact is in the EHL regime, the surfaces are still elastically deformed when this occurs.

To understand the link between the recovery of the roughness and the lift-off speed, the "real lambda" ratio calculated as the ratio between the average film thickness in the area of analysis and the in-contact RMS is shown Fig. 19. It can be seen that full surface separation occurs at a real lambda ratio $\lambda_{\text {Lift }} \approx 3.5$ for all three rough surfaces.

The fact that the surface separation occurs at a given value of the lambda ratio suggests that the transition between the mixed-lubrication and the full film regimes is

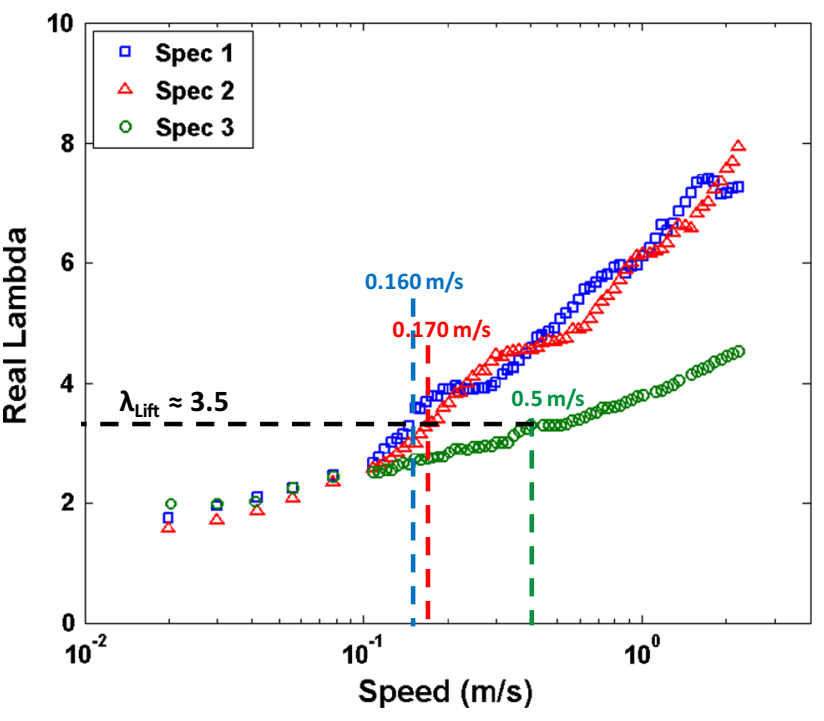

Fig. 19 Evolution with speed of the real lambda ratio in pure rolling conditions

mostly dominated by a balance between the roughness recovery and the lubricant film build-up in the contact.

\subsection{Calculated Pressure Distribution}

The film thickness maps of specimen 1 were used to estimate the pressure distribution variation with speed. Figure 20a shows a calculated pressure map in the static contact and illustrates the high pressure regions along the ridges. Figure $20 \mathrm{~b}$ compares a pressure profile transverse to the ridge direction in static conditions with the Hertz pressure for a smooth contact at the same load. Also shown in this figure is the corresponding normalised out-of-contact roughness profile (the actual amplitude of the asperities is about $0.5 \mu \mathrm{m}$ ). As expected, peaks of pressure can be observed at the asperity ridges.

On average, the pressure distribution seems to follow the Hertz distribution with local variations due to the presence of roughness. The highest pressure is observed in the centre of the contact, and the pressure drops in the outer parts. Figure 21a shows how the pressure evolves when the entrainment speed increases and the pressure builds up.

As the speed increase, the surfaces separate and they become less conformal. In the central area, this has the effect of redistributing the load support towards the top of the asperities that see the pressure rise, while it remains low and even diminishes in the valleys. At the outer edges, the pressure evolves in the opposite direction creating a low pressure "horseshoe" that can be seen in Fig. 21b similar to the one observe in a smooth contact. No pressure peak, known as the Petrusevich peak, could be seen at the exit, but it may be linked to the smoothing of the surfaces before they were used for the pressure calculations. 

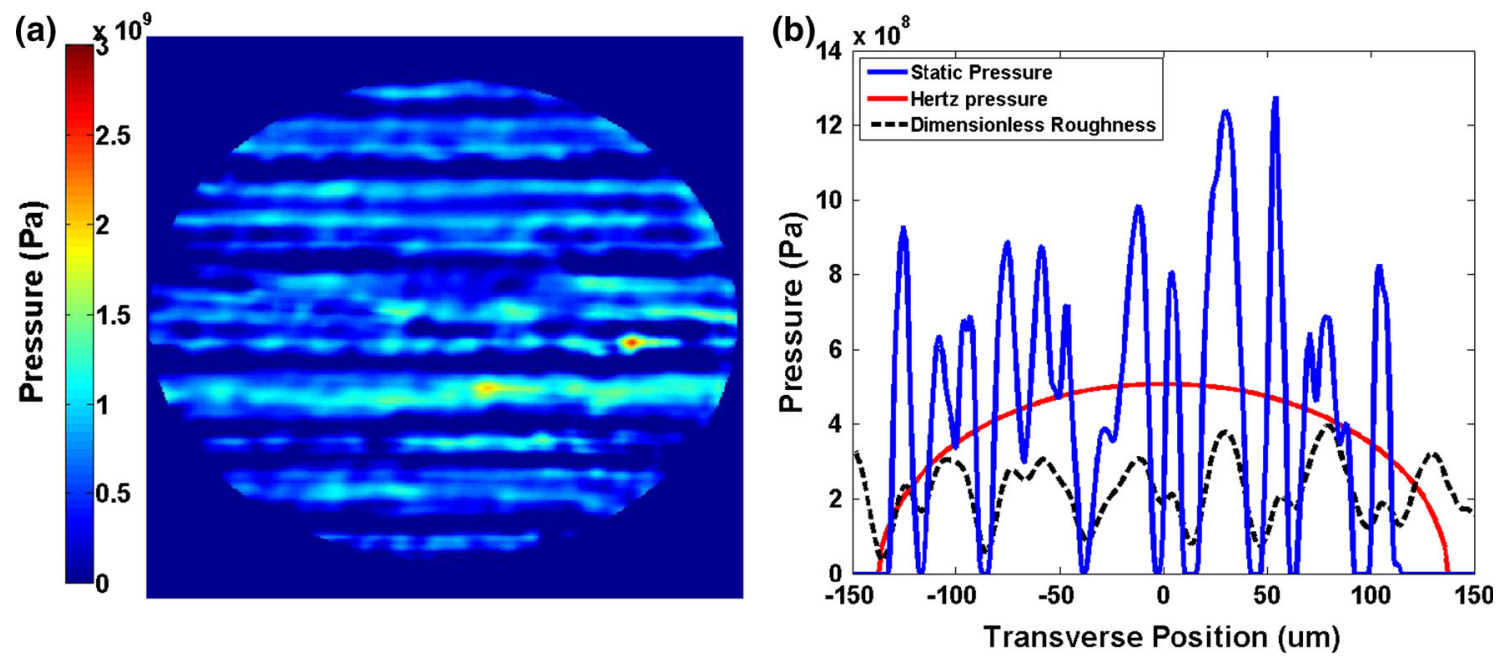

Fig. 20 a Pressure distribution over the contact, $\mathbf{b}$ comparison of the contact pressure in static conditions with the Hertz pressure, the roughness profile is also shown
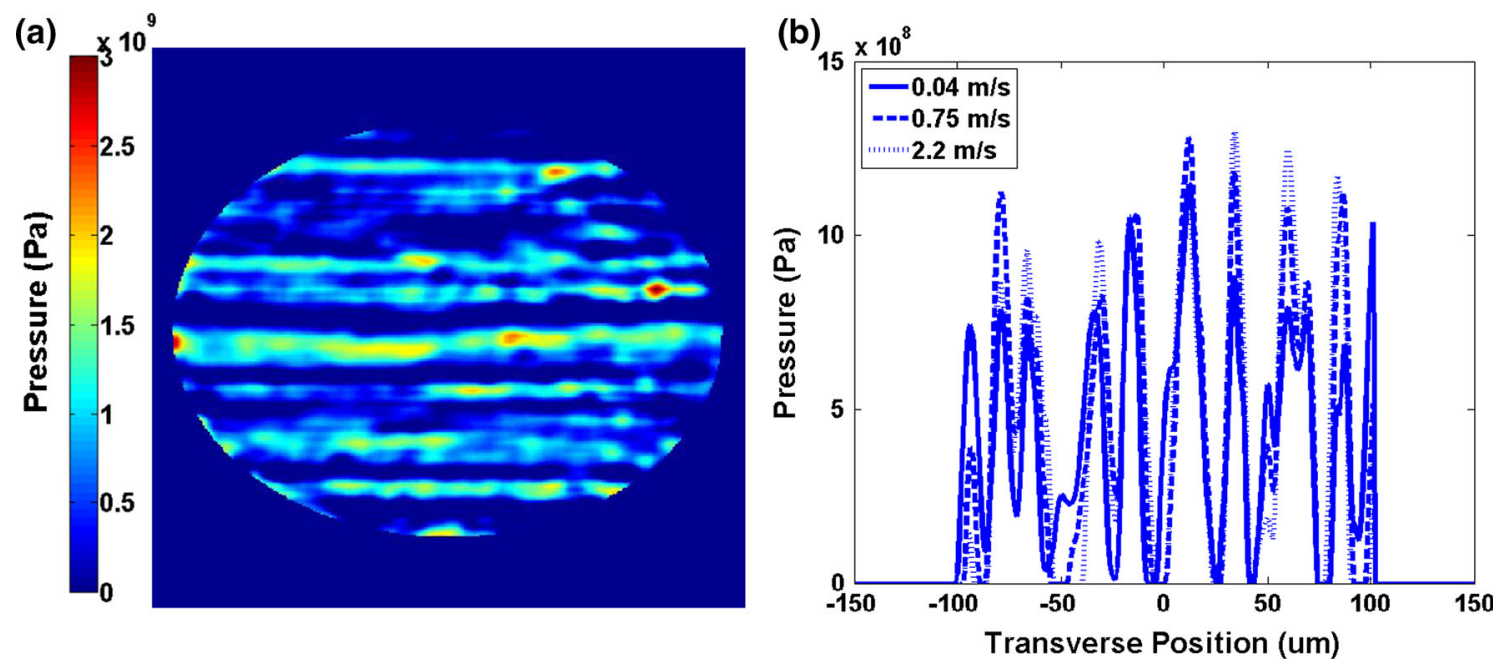

Fig. 21 a Pressure distribution at $2.2 \mathrm{~m} / \mathrm{s}$, b evolution with speed of the pressure profile in pure rolling conditions

To explain the evolution of pressures at the peaks and the valleys, an analogy with a smooth contact can be drawn. A smooth specimen was tested using the same lubricant and under the same operating conditions. Similar to the rough specimen, the film maps were used to obtain the pressure distribution at different speeds. In Fig. 22, the pressure distribution in the transverse direction is shown at three different speeds.

As expected, at low speeds the pressure profile is almost Hertzian. As the speed increases, the pressure at the edges decreases, this is due to the formation of the constriction. Interestingly, the pressure in the centre of the contact increases with speeds, as if the loss of load support in the horseshoe is compensated by an increase in load support in the central area. The reduction in bearing capacity at the sides and exit is accompanied by an increase in the pressure in the inlet. However, as mentioned previously, this does not affect the pressure distribution in the contact area. This pressure increase compared with the static case can be seen in other studies (see $[22,34]$ ) although this has not previously been formally identified.

For the rough case, if each ridge is considered as an individual micro-EHL contact, the same phenomenon would imply that as the speed increases, the pressure drops at the edges (i.e. the valleys) and increases in the centre of the contact area (i.e. the peaks), which is what is observed. The general horseshoe shape also seems to influence the pressure distribution as the pressure drop in the valleys located in the outer parts is much more dramatic than for the valleys located in the centre. 


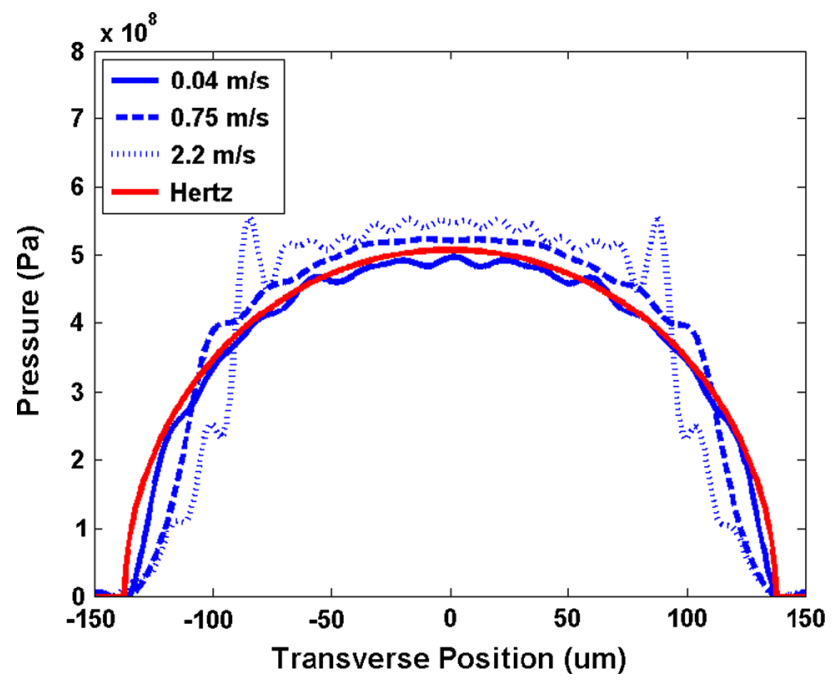

Fig. 22 Evolution with speed of the transverse pressure profile in the smooth case in pure rolling conditions

\subsection{Difference Between Longitudinal and Localised Roughnesses}

The results shown in this paper appear to contradict a number of previous studies that focused on localised roughness such as dents or transverse roughness. First, in these studies sliding was found to have an influence on the film thickness distribution, while such effects were not observed in the current study. For the case of transverse ridges, various authors $[1,3]$ have reported that, in the presence of sliding, a perturbation in the oil film was apparent when the ridges entered the contact. Analogous observations were made on dents (see $[12,35]$ ) or bumps [14]. Secondly, it has been found in this study that the roughness recovery was primarily influenced by the RMS roughness rather than the wavelength of the texture. Again, this seems to contradict previous theoretical work [17] that predicts the contrary.

When considering the influence of roughness on EHD film thickness, it is very important to realise that the primary impact of localised roughness features such as dents or transverse ridges occurs in the contact inlet region, where a roughness feature has the effect of momentarily changing the combined radius of the surfaces and thus the rate at which the film gap converges. This causes a transient change in the quantity of lubricant being entrained and thus produces a momentary variation in film thickness. This "blip" or more formally "perturbation" in the film thickness then passes through the contact at the mean speed of the surfaces.

However, for longitudinal roughness, where the surface features are elongated precisely along the entrainment direction this does not occur. The ridges and valleys both have almost the same radius along the rolling/sliding direction as does the overall surface, so the system is essentially a steady-state one. In this case, uniquely, the main impact of the roughness occurs within the contact itself and results from the responses of the fluid to the local conditions generated by the varying pressure field therein, for example to the very high pressure along the ridges and to the difference in pressure between the fluid at the ridges and in the valleys. These differences in studied contacts may at least in part explain apparent discrepancies between results shown here and those found by previous studies.

This fundamental difference between longitudinal and all other forms of roughness explains why the results shown are quite different from that found by many previous authors.

\section{Conclusions}

In this study, a novel procedure to enable film thickness mapping in rough surface EHL contacts has been presented. The study uses steel balls with real machined surface roughnesses characterised by ridges oriented parallel to the entrainment direction. Film thickness measurements have been conducted under pure rolling and rolling-sliding conditions over a wide speed range such that it is possible to observe the progression from mixed/boundary to full film EHL. Film thickness maps from the central region of the rough contact have been obtained and the film distribution compared to the classic, smooth case equivalent.

The results show:

- Minimum film thickness, observed at the top of the ridges, is much smaller than film thickness in smooth contact.

- The transition from mixed to full film lubrication occurs at higher speeds than for a smooth surface.

- An average film thickness in rough contacts is very similar to the smooth case.

- A very large reduction in the effective composite roughness at low entrainment speed is followed by a recovery when the lubricant film starts to build up.

The three specimens tested were selected to assess the respective influence of the dominant roughness wavelength and the RMS roughness on the development of an EHL film. In the conditions tested, the only parameter that had a significant influence was the RMS roughness of the specimen, which affected the lift-off speed and the minimum film thickness. The influence of sliding on the film build-up was found to be negligible at the sliding speeds considered.

Micro-EHL films were observed to develop at the ridges. With increasing speed, a macro-EHL horseshoe shape was eventually formed, analogous to that seen with smooth 
surfaces. It was found that, at the highest speeds studied, the macro-scale deformation of the rough surface matched the deformation observed on a smooth ball at the same conditions.

The maps of film thickness of one specimen were used to calculate the deformation field which was then subject to an inverse solution model to calculate the pressure field. Counter-intuitively, the pressure at the peaks increased with speed, while it decreases in the valley. This phenomenon was explained by the formation of a micro-EHL film at the top of the ridges.

Finally, the surface lift-off, and hence the transition between mixed/lubrication and full EHL regime, was shown to be closely linked to the average film thickness in the contact and the roughness recovery.

Acknowledgments The authors would like to thank Dr. Antonio Gabelli for providing the rough ball specimens and SKF BV for sponsoring this research through the SKF University Technology Centre for Tribology at Imperial College London.

Open Access This article is distributed under the terms of the Creative Commons Attribution 4.0 International License (http:// creativecommons.org/licenses/by/4.0/), which permits unrestricted use, distribution, and reproduction in any medium, provided you give appropriate credit to the original author(s) and the source, provide a link to the Creative Commons license, and indicate if changes were made.

\section{References}

1. Kaneta, M., Sakai, T., Nishikawa, H.: Effects of surface roughness on point contact EHL. Tribol. Trans. 36, 605-612 (1993)

2. Guangteng, G., Cann, P.M., Olver, A.V., Spikes, H.A.: Lubricant film thickness in rough surface, mixed elastohydrodynamic contact. J. Tribol. 122, 65-76 (2000)

3. Choo, J.W., Olver, A.V., Spikes, H.A.: The influence of transverse roughness in thin film, mixed elastohydrodynamic lubrication. Tribol. Int. 40, 220-232 (2007)

4. Christensen, H.: Stochastic models for hydrodynamic lubrication of rough surfaces. Proc. Inst. Mech. Eng. 184, 1013-1026 (1970)

5. Johnson, K.L., Greenwood, J.A., Poon, Y.: A Simple Theory of Asperity Dynamic Lubrication Contact in Elastohydrodynamic Lubrication. Wear 19, 91-108 (1972)

6. Janczak, K.J., Wisniewski, M.R.: Influence of surface roughness parameters on elastohydrodynamic film thickness. Wear 115, 75-82 (1987)

7. Cameron, A., Gohar, R.: Theoretical and experimental studies of the oil film in lubricated point contact. Proc. R. Soc. A Math. Phys. Eng. Sci. 291, 520-536 (1966)

8. Jackson, A., Cameron, A.: An interferometric study of the EHL of rough surfaces. ASLE Trans. 19, 50-60 (1976)

9. Wedeven, L.D., Cusano, C.: Elastohydrodynamic film thickness measurements of artificially produced surface dents and grooves. ASLE Trans. 22, 369-381 (1979)

10. Dumont, M.-L., Lugt, P.M., Tripp, J.H.: Surface feature effects in starved circular EHL contacts. J. Tribol. 124, 358-366 (2002)

11. Ren, N., Nanbu, T., Yasuda, Y., Zhu, D., Wang, Q.: Micro textures in concentrated-conformal-contact lubrication: effect of distribution patterns. Tribol. Lett. 28, 275-285 (2007)
12. Mourier, L., Mazuyer, D., Lubrecht, A.A., Donnet, C.: Transient increase of film thickness in micro-textured EHL contacts. Tribol. Int. 39, 1745-1756 (2006)

13. Kaneta, M., Nishikawa, H.: Experimental study on microelastohydrodynamic lubrication. Proc. Inst. Mech. Eng. Part J J. Eng. Tribol. 213, 371-381 (1999)

14. Choo, J.W., Glovnea, R.P., Olver, A.V., Spikes, H.A.: The effects of three-dimensional model surface roughness features on lubricant film thickness in EHL contacts. J. Tribol. 125, 533-542 (2003)

15. Greenwood, J.A., Johnson, K.L.: The behaviour of transverse roughness in sliding elastohydrodynamic lubricated contacts. Wear 153, 107-117 (1992)

16. Choo, J.W., Olver, A.V., Spikes, H.A., Dumont, M.-L., Ioannides, E.: The influence of longitudinal roughness in thin-film, mixed elastohydrodynamic lubrication. Tribol. Trans. 49, 248-259 (2006)

17. Venner, C.H., Morales-Espejel, G.E.: Amplitude reduction of small amplitude waviness in transient elastohydrodynamically lubricated line contacts. Proc. Inst. Mech. Eng. Part J J. Eng. Tribol. 213, 487-504 (1999)

18. Jacod, B., Lugt, P.M., Dumont, M.-L., Tripp, J.H., Venner, C.H.: Amplitude reduction of waviness in elastohydrodynamic lubrication using an eyring fluid model. Proc. Inst. Mech. Eng. Part J J. Eng. Tribol. 214, 343-350 (2000)

19. Venner, C.H., Lubrecht, A.A.: Amplitude Reduction of Nonisotropic Harmonic Patterns in Circular EHL Contacts Under Pure Rolling. Tribology Series, vol. 36, pp. 151-162 (1999)

20. Masen, M.A., Venner, C.H., Lugt, P.M., Tripp, J.H.: Effects of surface micro-geometry on the lift-off speed of an EHL contact. Tribol. Trans. 45, 21-30 (2002)

21. Astrom, H., Venner, C.H.: Soap-thickener induced local pressure fluctuations in a grease-lubricated elastohydrodynamic point contact. Proc. Inst. Mech. Eng. Part J J. Eng. Tribol. 208, 191-198 (1994)

22. Molimard, J., Querry, M., Vergne, P., Krupka, I., Hartl, M.: Calculation of pressure distribution in EHD point contacts from experimentally determinated film thickness. Tribol. Int. 38, 391-401 (2005)

23. Love, A.E.H.: The stress produced in a semi-infinite solid by pressure on part of the boundary. Philos. Trans. R. Soc. 228, 377-420 (1929)

24. Stanley, H.M., Kato, T.: An FFT-based method for rough surface contact. J. Tribol. 119, 481-485 (1997)

25. Kadiric, A., Sayles, R.S., Ioannides, E.: Thermo-mechanical model for moving layered rough surface contacts. J. Tribol. 130, 1-14 (2008)

26. Spikes, H.A., Cann, P.M.: The development and application of the spacer layer imaging method for measuring lubricant film thickness. Proc. Inst. Mech. Eng. Part J J. Eng. Tribol. 215, 261-277 (2001)

27. Molimard, J.: Etude Experimentale du Regime de Lubrification en Film Mince - Application aux Fluides de Laminage. PhD Thesis, Insa Lyon (1999)

28. Wedeven, L.D.: Optical Measurements in Elastohydrodynamic Rolling-Contact Bearings. PhD Thesis, Univ. London (1970)

29. Kaneta, M., Tani, N., Nishikawa, H.: Optical Interferometric Observations of the Effect of Moving Transverse Asperities on Point Contact EHL Films. Tribology Series, vol. 41, pp. 101-109 (2003)

30. Hartl, M., Krupka, I., Poliscuk, R., Liska, M.: An automatic system for real-time evaluation of EHD film thickness and shape based on the colorimetric interferometry. Tribol. Trans. 42, 303-309 (1999)

31. Choo, J.W., Olver, A.V., Spikes, H.A.: Influence of surface roughness features on mixed film lubrication. Lubr. Sci. 15, 219-232 (2003) 
32. Johnston, G.J., Wayte, R., Spikes, H.A.: The measurement and study of very thin lubricant films in concentrated contacts the measurement and study of very thin lubricant films in concentrated contacts. Trib. Trans. 34, 187-194 (1991)

33. Luo, J., Wen, S., Huang, P.: Thin film lubrication. Part I. Study on the transition between EHL and thin film lubrication using a relative optical interference intensity technique. Wear 194, 107-115 (1996)
34. Höhn, B.R., Michaelis, K., Kreil, O.: Influence of surface roughness on pressure distribution and film thickness in EHLcontacts. Tribol. Int. 39, 1719-1725 (2006)

35. Krupka, I., Hartl, M.: Experimental study of microtextured surfaces operating under thin-film EHD lubrication conditions. J. Tribol. 129, 502-508 (2007) 